\title{
Constitutive expression of suppressor of cytokine signalling-3 in skeletal muscle leads to reduced mobility and overweight in mice
}

\author{
P. Lebrun • E. Cognard • R. Bellon-Paul • P. Gontard • C. Filloux • C. Jehl-Pietri • \\ P. Grimaldi • M. Samson • L. Pénicaud • J. Ruberte • T. Ferre • A. Pujol • F. Bosch • \\ E. Van Obberghen
}

Received: 16 April 2009 / Accepted: 22 June 2009/Published online: 12 August 2009

(C) Springer-Verlag 2009

\begin{abstract}
Aims/hypothesis Due to their ability to regulate various signalling pathways (cytokines, hormones, growth factors), the suppressor of cytokine signalling (SOCS) proteins are thought to be promising therapeutic targets for metabolic and inflammatory disorders. Hence, their role in vivo has to be precisely determined.

Methods We generated transgenic mice constitutively producing SOCS-3 in skeletal muscle to define whether the
\end{abstract}

P. Lebrun and E. Cognard contributed equally to this work.

Electronic supplementary material The online version of this article (doi:10.1007/s00125-009-1474-9) contains supplementary material, which is available to authorized users.

P. Lebrun $(\bowtie) \cdot$ E. Cognard $\cdot$ R. Bellon-Paul $\cdot$ P. Gontard

C. Filloux $\cdot$ C. Jehl-Pietri $\cdot$ P. Grimaldi $\cdot$ E. Van Obberghen $(\triangle)$

INSERM, Unit 907,

Avenue de Valombrose,

06107 Nice, France

e-mail: plebrun@unice.fr

e-mail: vanobbeg@unice.fr

P. Lebrun · E. Cognard · R. Bellon-Paul · P. Gontard • C. Filloux •

C. Jehl-Pietri · P. Grimaldi • M. Samson • E. Van Obberghen

Faculté de Médecine, Institut de Génétique et Signalisation

Moléculaire (IFR50), Université de Nice-Sophia Antipolis,

Nice, France

L. Pénicaud

CNRS-UPS, UMR 5241, Université Paul Sabatier,

Toulouse, France

J. Ruberte $\cdot$ T. Ferre $\cdot$ A. Pujol $\cdot$ F. Bosch

Center of Animal Biotechnology and Gene Therapy, Edifici H,

Universitat Autonoma de Barcelona,

Bellaterra, Spain sole abundance of SOCS-3 is sufficient to induce metabolic disorders and whether SOCS-3 is implicated in physiological roles distinct from metabolism.

Results We demonstrate here that chronic expression of SOCS-3 in skeletal muscle leads to overweight in mice and worsening of high-fat diet-induced systemic insulin resistance. Counter-intuitively, insulin sensitivity in muscle of transgenic mice appears to be unaltered. However, following constitutive SOCS-3 production, several genes had deregu-
J. Ruberte $\cdot$ F. Bosch

Department of Biochemistry and Molecular Biology,

School of Veterinary Medicine,

Universitat Autonoma de Barcelona,

Bellaterra, Spain

T. Ferre $\cdot$ A. Pujol

Department of Animal Health and Anatomy,

School of Veterinary Medicine,

Universitat Autonoma de Barcelona,

Bellaterra, Spain

J. Ruberte $\cdot$ T. Ferre $\cdot$ A. Pujol $\cdot$ F. Bosch

CIBER de Diabetes y Enfermedades Metabolicas Asociadas

(CIBERDEM),

Madrid, Spain

E. Van Obberghen

Laboratoire de Biochimie, Hôpital Pasteur,

CHU de Nice,

Nice, France 
lated expression, among them other members of the SOCS family. This could maintain the insulin signal into skeletal muscle. Interestingly, we found that SOCS-3 interacts with calcineurin, which has been implicated in muscle contractility. In Socs-3 transgenic muscle, this leads to delocalisation of calcineurin to the fibre periphery. Relevant to this finding, Socs-3 transgenic animals had dilatation of the sarcoplasmic reticulum associated with swollen mitochondria and decreased voluntary activity.

Conclusions/interpretation Our results show that constitutive SOCS-3 production in skeletal muscle is not in itself sufficient to induce the establishment of metabolic disorders such as diabetes. In contrast, we reveal a novel role of SOCS-3, which appears to be important for muscle integrity and locomotor activity.

Keywords Exercise - Insulin resistance - Overweight .

Skeletal muscle $\cdot$ SOCS $\cdot$ Transgenic mice

$\begin{array}{ll}\text { Abbreviations } \\ \text { CIS } & \text { Cytokine-inducible SH2-containing protein } \\ \text { CNTF } & \text { Ciliary neurotrophic factor (CNTF) } \\ \text { DHPR } & \text { Dihydropyridine-sensitive L-type calcium channel } \\ \text { EDL } & \text { Extensor digitorum longus } \\ \text { GSK-3 } & \text { Glycogen synthase kinase-3 } \\ \text { GST } & \text { Glutathione S-transferase } \\ \text { HFD } & \text { High-fat diet } \\ \text { IPGTT } & \text { Intra-peritoneal glucose tolerance test } \\ \text { IPITT } & \text { Intra-peritoneal insulin tolerance test } \\ \text { MLC } & \text { Myosin light chain } \\ \text { PKB } & \text { Protein kinase B } \\ \text { r-t PCR } & \text { real-time PCR } \\ \text { RyR } & \text { Ryanodine receptors } \\ \text { SDH } & \text { Succinate dehydrogenase } \\ \text { SOCS } & \text { Suppressor of cytokine signalling }\end{array}$

\section{Introduction}

Suppressor of cytokine signalling (SOCS) proteins are feedback inhibitors of signalling pathways induced by a wide panel of stimuli, including hormones, cytokines and growth factors. This family of regulators is composed of SOCS-1 to SOCS-7 and the cytokine-inducible SH2containing protein (CIS) [1]. Upon induction by various stimuli, SOCS protein levels rapidly increase, but content is only transiently augmented. First revealed as repressors of cytokine signalling, SOCS proteins have been shown to be also potent inhibitors of hormone-induced signalling. In brief, we and others have reported that SOCS-3 produced in response to insulin attenuates subsequent propagation of insulin signalling by hampering binding and phosphorylation of IRSs [2, 3] and by targeting IRS-1 and IRS-2 for proteasomal degradation [4]. The impact of SOCS-3 on metabolism can also occur via inhibition of leptin signalling, which regulates food intake, energy balance and neuroendocrine functions $[5,6]$.

Several mouse models have been generated to investigate the role of SOCS-3 in vivo. Thus, Socs-3-deficient mice demonstrated the implication of SOCS-3 in regulating leptin signalling pathways in neurons [7, 8]. Moreover, SOCS-3 constitutive production in liver decreased IRS tyrosine phosphorylation [3] and induced systemic insulin resistance and hepatic steatosis [9, 10]. However, using transgenic mice constitutively producing SOCS-3 in adipose tissue, it has been shown that SOCS-3 decreases insulin signalling in adipose tissue, but this is insufficient to induce organismal hormone resistance [11]. Finally, a study of transgenic mice producing high levels of SOCS-3 in pancreatic beta cells suggests a role of SOCS-3 in controlling growth hormone and cytokine pathways in vivo $[12,13]$. While collectively these data suggest that SOCS-3 profoundly affects cell physiology, the signalling pathways modulated by SOCS-3 appear to vary from one tissue to another.

Despite the key role of skeletal muscle in the control of organismal glucose homeostasis, the role of SOCS-3 in this tissue has been studied mainly using cultured cells. Indeed, it was shown that SOCS-3 expression can be induced by IGF-1 during C2C12 myoblast differentiation [14]. SOCS-3 can also attenuate insulin-stimulated glycogen synthesis in L6 myotubes [3]. In vivo, abundance of SOCS-3 in skeletal muscle has been shown in several pathophysiological conditions such as exercise, insulin resistance and obesity $[3,15]$. However, it is still a matter of debate whether augmented levels of SOCS-3 is a cause or a consequence of insulin resistance.

Interestingly, SOCS-3 production can be induced by catecholamines [16], which play a crucial role in muscle contraction. In addition, the ability of SOCS-3 to interact with calcineurin and to hamper its downstream signalling has been reported [17]. Even though these results were obtained in a system different from myocytes, it is important to note that fluxes of $\mathrm{Ca}^{2+}$, necessary for skeletal muscle functioning, are tightly regulated by calcineurin [18].

Collectively, most of the data would suggest that SOCS3 plays an important role in regulation of skeletal muscle metabolism. Hence, we generated transgenic mice constitutively producing SOCS-3 in skeletal muscle to investigate whether the sole abundance of SOCS-3 is sufficient to induce metabolic disorders and whether SOCS-3 plays physiological roles beyond regulation of metabolism. 


\section{Methods}

Materials

Rabbit antibodies to IRS- 1 and IRS-2 used for immunoprecipitation were home-made and from Upstate Biotechnology (Lake Placid, NY, USA), respectively. Antibodies to SOCS-3 and glycogen synthase kinase-3 (GSK-3) $\beta$ were purchased from Santa Cruz Biotechnology (Santa Cruz, CA, USA) and those to phospho-GSK-3 $\alpha / \beta$ (Ser21/9), protein kinase B (PKB) (aa 466-473) and phospho-PKB (Ser 473) were from Cell Signaling Technology (Danvers, MA, USA). Antibodies to p85 and IRS-1 for western blotting were from Upstate Biotechnology. The anticalcineurin (Pan A polyclonal antibody, AB 1695) was from Chemicon (Millipore, Billerica, MA, USA). For all experiments, we used human insulin (Insuman Rapid; Aventis Pharma Deutschland, Frankfurt, Germany).

Generation of transgenic myosin light chain/Socs-3 mice

The myosin-light chain (MLC) 1 promoter/enhancer has been previously used for restricted expression in skeletal muscle $[19,20]$. Briefly, a flag-tagged $0.7 \mathrm{~kb}$ EcoRI-EcoRI fragment containing the entire coding sequence of mouse Socs-3 cDNA (from D. Hilton, The Walter and Eliza Hall Institute of Medical Research, VIC, Australia) was introduced in pMDAF2-MLC1. Our mice had a C57BL/6 background. Care of animals was performed in accordance with the Guidelines for the care and use of laboratory animals of the National Institute of Health and Medical Research of France (INSERM, France).

RNA extraction, reverse transcription and real-time PCR

Frozen tissues were homogenised in Trizol (Invitrogen Life Technologies, Gaithersburg, MD, USA), and RNAs were extracted and reverse-transcribed (AMV-RT; Promega, Madison, WI, USA). cDNAs were analysed using SYBR Green real-time PCR (ABI PRISM 7000 Sequence Detector System, Applied Biosystems, Foster City, CA, USA). The amount of cDNA used in each reaction was normalised to housekeeping $36 b 4$ (also known as Rplp0) cDNA.

Animal studies and metabolic analysis

Mice were housed on a $12 \mathrm{~h}$ light/dark cycle and placed at 5 weeks old on a high-fat diet (HFD) (42\% fat, TD-88137; Harlan Teklab, Madison, WI, USA) or standard chow diet with free access to diet and water. Body weight and food intake were measured weekly. For intra-peritoneal glucose tolerance test (IPGTT) and intra-peritoneal insulin tolerance tests (IPITT), $2 \mathrm{mg} / \mathrm{g}$ body weight and $0.75 \mathrm{mU} / \mathrm{g}$ body weight of glucose and insulin, respectively, were injected intraperitoneally. Glycaemia was measured using a glucometer (One Touch; Lifescan, Milpitas, CA, USA).

Ex vivo insulin signal in isolated muscles

Stimulation of intact extensor digitorum longus (EDL) muscle ex vivo with insulin $(1 \mathrm{nmol} / \mathrm{l})$ was done in KrebsRinger buffer as previously described [21]. For protein extraction, tissues were lysed as previously described [22] and proteins were quantified (BCA protein assay kit; Pierce/Thermo Scientific, Rockford, IL, USA), separated by SDS-PAGE, transferred to PVDF membranes and blotted with antibodies. Immunoreactive proteins were revealed by enhanced chemiluminescence.

\section{Microarray}

cDNA was generated from 300 ng of total RNA using a kit (GeneChip WT cDNA Synthesis and Amplification Kit; Affymetrix, Santa Clara, CA, USA) and was fragmented and end-labelled using the Terminal Labeling Kit (GeneChip WT; Affymetrix). Labelled DNA targets were hybridised to the Affymetrix GeneChip Mouse Gene 1.0 ST Array at $45^{\circ} \mathrm{C}$ for $17 \mathrm{~h}$, according to manufacturer's recommendations. Hybridised arrays were washed and stained on a GeneChip Fluidics Station 450 and scanned on a GeneChip Scanner 3000 7G (Affymetrix). Gene expression levels were estimated using Gene Level-RMA sketch method in Expression Console software (Affymetrix). Normalised data were then analysed with the lima package from Bioconductor [23]. Microarray data are archived in GEO website (www.ncbi.nlm.nih.gov/ geo/query/acc.cgi?token=jpwtxqieaucgihm\&acc $=$ GSE17063, accessed 20 July 2009).

Glutathione $S$-transferase pull-down and proteomics

For glutathione $S$-transferase (GST) pull-down experiments, muscle lysates were incubated with GST or GST/SOCS-3 beads for $75 \mathrm{~min}$ at room temperature. Interacting proteins were separated by SDS-PAGE and analysed by western blotting or proteomics [24].

Histological analysis

Staining for succinate dehydrogenase (SDH) has been described elsewhere [25]. Cryosections were cut from the middle portion of frozen tibialis anterior muscle. The number of SDH-positive fibres (moderately or darkly stained) was counted in whole-muscle sections.

For transmission electron microscopic analysis, tibialis anterior muscle fragments were fixed in $2.5 \%$ (vol./vol.) glutaraldehyde and $2 \%$ (vol./vol.) paraformaldehyde, post- 
fixed in $1 \%$ (vol./vol.) osmium tetroxide, stained in aqueous uranyl acetate, dehydrated and embedded in epoxy resin. Ultrathin sections $(70 \mathrm{~nm})$ were stained using lead citrate and examined by transmission electron microscopy (H-7000; Hitachi, Schaumburg, IL, USA).

For immunohistochemistry, the following antibodies were used: rabbit anti-human calcineurin antibody (1:100) and goat anti-mouse SOCS-3 antibody (1:100) (both from Santa Cruz Biotechnology); biotinylated anti-rabbit IgG (Vector Laboratories, Burlingame, CA, USA); and streptavidin Alexa Fluor 488 and anti-goat Alexa Fluor 568 (both from Molecular Probes). ToPro3 iodide (Molecular Probes/ Invitrogen/Life Technologies, Carlsbad, CA, USA) for nuclear counterstaining was used for laser-scanning confocal analysis (TCS SP2; Leica Microsystems, Wetzlar, Germany).

\section{Indirect calorimetry and locomotor activity}

The following metabolic variables were measured on individually-caged mice (Oxylet, Panlab-Bioseb, Chaville, France): oxygen consumption $\left(\dot{V} \mathrm{O}_{2}\right)$, carbon dioxide production $\left(\dot{V} \mathrm{CO}_{2}\right)$, energy expenditure and locomotor activity [26]. Measurements were performed over a $24 \mathrm{~h}$ period and data were averaged for each mouse. Physical activities of the mice were monitored by an infrared photocell beaminterruption method (Panlab-Bioseb, Chaville, France). For voluntary exercise, mice were individually housed in cages equipped with a $25 \mathrm{~cm}$ diameter wheel. The counter connected to the wheel counts the number of run quarters and was reset every day.

\section{Statistical analysis}

Results are presented as means \pm SEM. $n$ represents the number of mice. Differences between groups were compared with the two-tailed unpaired Student's $t$ test. A $p$ value of $\leq 0.05$ was considered significant.

\section{Results}

Constitutive production of SOCS-3 in skeletal muscle of MLC/Socs-3 mice

SOCS protein level is very low under basal conditions. As expected, SOCS-3 protein was detectable by western blot exclusively in transgenic skeletal muscle (Fig. 1a). We verified by real-time PCR that Socs-3 mRNA was constitutively expressed only in skeletal muscle, but not in other muscles of our transgenic mice (Fig. 1b). Expression of the mRNA transgene is high in glycolytic and mixed muscles, but less pronounced in the oxidative soleus muscle (Fig. 1c). Our transgenic mice had normal fertility and a Mendelian transgene distribution.

Metabolic study of MLC/Socs-3 mice

Weight and adipose mass Weight of transgenic and wildtype mice was monitored for 14 weeks. As shown in Fig. 2a, transgenic mice at 8 to 13 weeks of diet had a weight gain significantly higher than that observed for wild-type mice, both on standard chow and on HFD. Next, we studied the adiposity of our mice and demonstrated that transgenic mice, after 14 weeks on HFD, had increased perigonadal fat mass (Fig. 2b) (for cellular adiposity see Electronic supplementary material [ESM] Fig. 1). This is representative of total white adipose mass, which doubled in transgenic animals on HFD, whereas brown adipose tissue mass was unchanged (data not shown).

Food intake and energy expenditure in MLC/Socs-3 mice Measurement of energy intake indicated that from 8 to 14 weeks of diet (chow and HFD) transgenic mice seemed to eat more than wild-type mice (Fig. 2c). However, energy intake normalised for the mean weight of each group of animals (Fig. 2d) suggested that the greater food
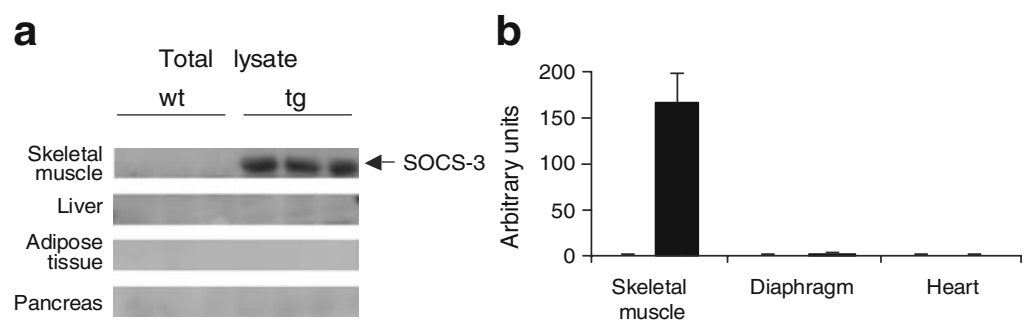

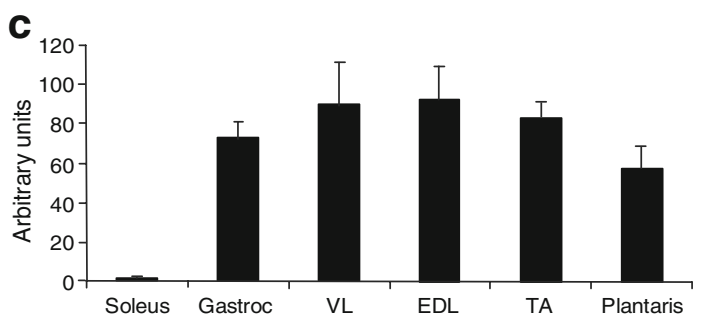

Fig. 1 Generation of transgenic mice constitutively producing SOCS3 in skeletal muscle. a Total extracts from insulin-sensitive tissues (as indicated) were prepared from wild-type (wt) and transgenic ( $\mathrm{tg}$ ) mice and analysed by western blot with antibody to SOCS-3. b Socs-3 transgene expression is restricted to skeletal muscle. Heart, diaphragm and skeletal muscles were isolated from transgenic (black bars) and wild-type (white bars) mice $(n=2)$. RNA was extracted and analysed by real-time PCR. c Different types of skeletal muscle (soleus, gastrocnemius [gastroc], vastus lateralis [VL], EDL, tibialis anterior [TA] and plantaris) were isolated from transgenic mice $(n=3)$ and analysed as above (b) 
Fig. 2 a, b MLC/Socs-3 transgenic mice have increased body weight and enlarged fat mass compared with wild-type littermates on standard chow and HFD. Mice on chow diet (wildtype, $n=7$, white squares; transgenic, $n=7$, black diamonds) or on HFD (wild-type, $n=7$, white circles; transgenic, $n=6$, black triangles) were weighed once a week, starting at 5 weeks of age and for 14 weeks (a). Perigonadal adipose tissue (b) was isolated from wild-type (white bars) and transgenic (black bars) mice at 24 weeks of age (chow diet, $n=5$ per mouse type) and at 25 weeks of age (HFD, $n=4$ per group), and weighed. c, d Similar energy intake in MLC/ Socs3 transgenic mice compared with wild-type littermates. Food was weighed once a week and converted to energy value $(\mathrm{kJ})$. An average per mouse and per day was calculated for each group (c). Food intake (kJ) was normalised to body weight (BW, $\mathrm{g}$ of BW) for each group (d). $n=$ 5 for each group. e, f Reduced energy expenditure in MLC/ SOCS-3 transgenic mice compared with wild-type littermates e Oxygen consumption $\left(\dot{V} \mathrm{O}_{2}\right)$ and (f) energy expenditure (EE) were measured during $24 \mathrm{~h}$ by indirect calorimetry in 11-week-old males on chow diet (wild-type, white bars, $n=7$; transgenic, black bars, $n=6$ ) $* p \leq 0.05$
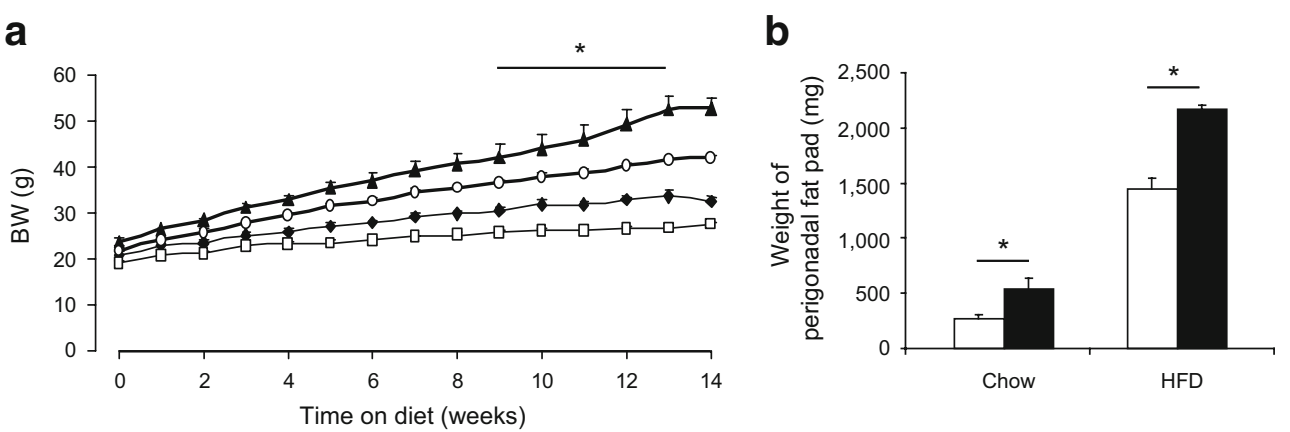

C

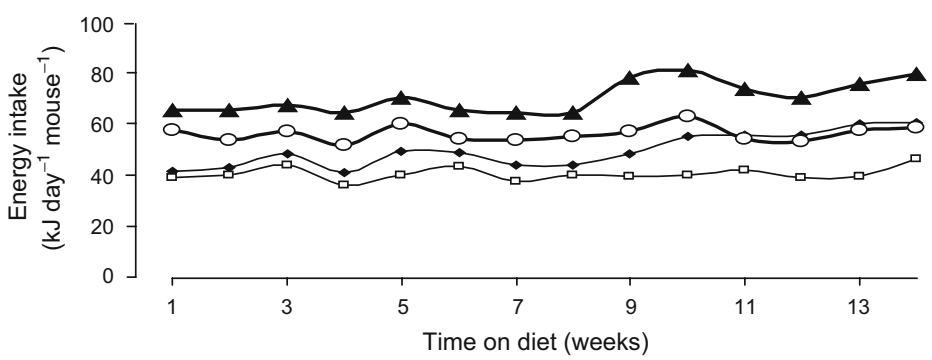

d

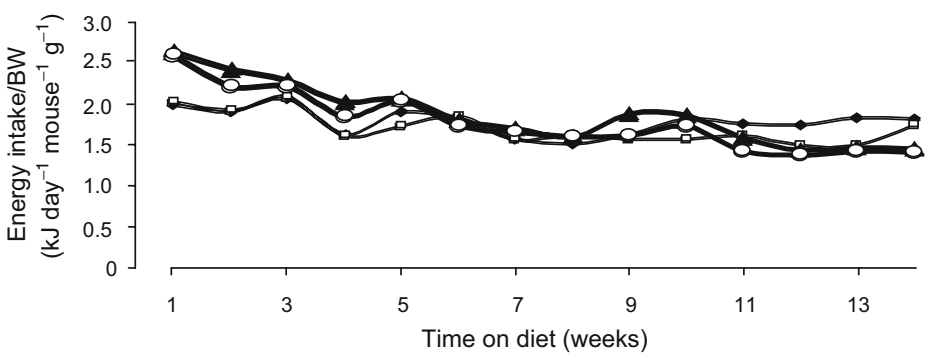

e

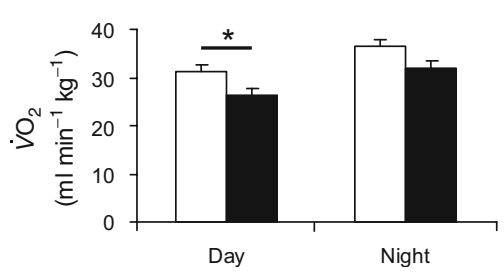

f

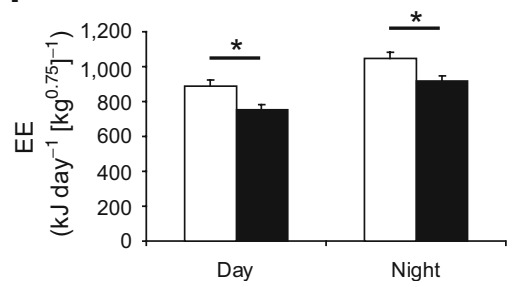

intake in transgenic mice reflected their increased body mass. Thus, despite a similar energy intake per g body weight, the MLC/Socs-3 mice gained more weight than their wild-type littermates.

Since the two major factors regulating weight gain are food intake and energy expenditure, we next analysed whether our transgenic mice had altered energy expenditure. As shown in Fig. 2e, the $\dot{V} \mathrm{O}_{2}$ intake was reduced by $16 \%$ and $12 \%$ in transgenic mice during day and night, respectively. The decrease in $\dot{V} \mathrm{O}_{2}$ calculated during the night was not statistically significant $(p=0.07)$. Compatible with the $\dot{V} \mathrm{O}_{2}$ measurements, we observed reduced energy expenditure in our transgenic mice both during day and night (15.5\% and $12.8 \%$, respectively) (Fig. 2f). These results strongly suggest that the fat accumulation observed in MLC/Socs-3 mice could be due to altered energy expenditure rather than to increased food intake.

Insulin and glucose tolerance We performed IPGTT and IPITT to define whether the transgenic mice were able to properly regulate their glycaemia and whether they were insulin-resistant.

Using IPGTT, we found that MLC/Socs-3 mice had normal glucose tolerance (Fig. 3a). However, regulation of glycaemia in response to insulin was altered in MLC/Socs-3 


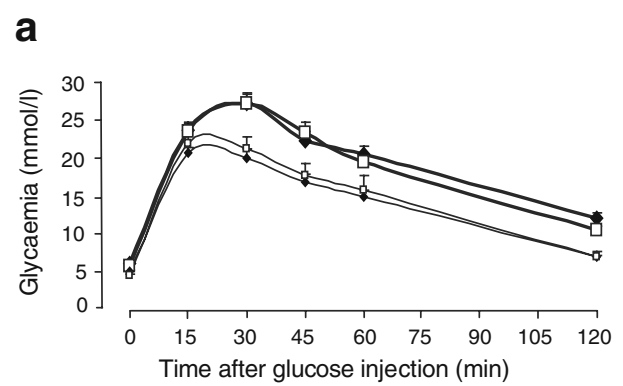

C

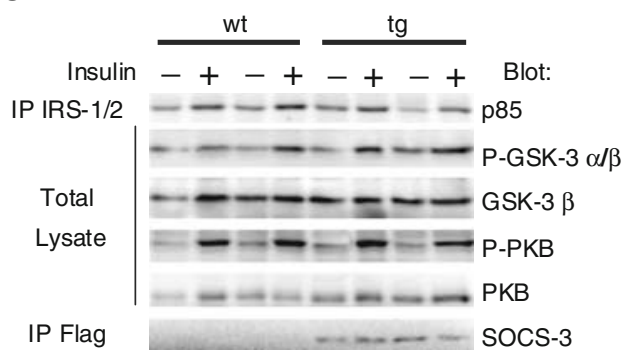

e

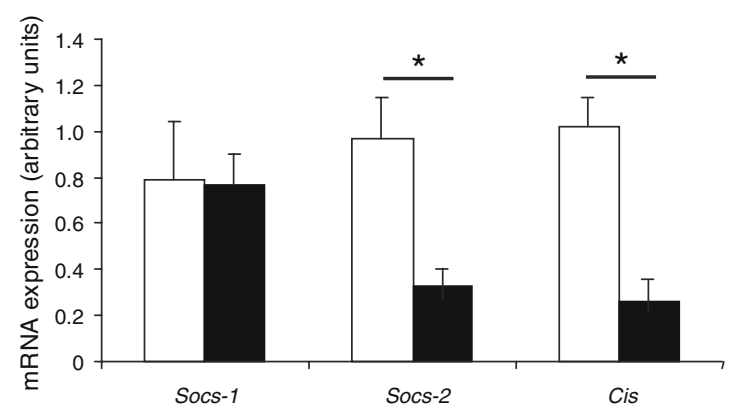

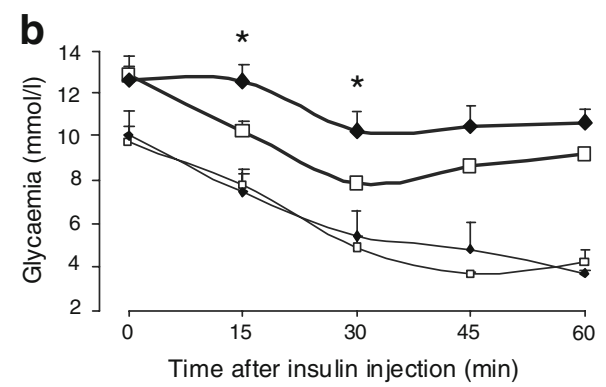

d

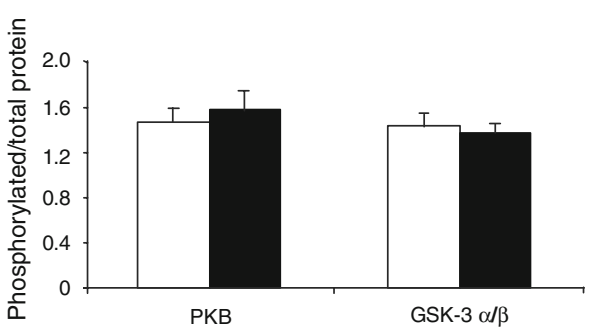

f

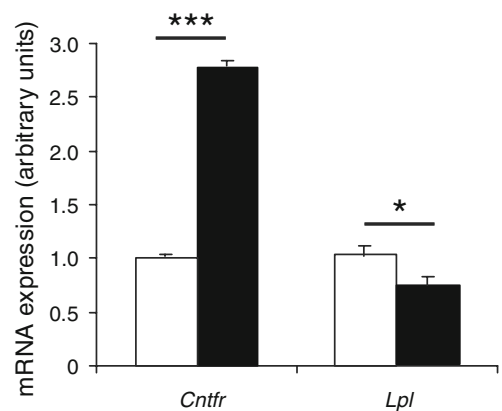

Fig. 3 a, b Metabolic study in MLC/Socs-3 transgenic mice. IPGTT (a) and IPITT (b) in MLC/Socs-3 transgenic mice on chow (black diamonds) and HFD (large black diamonds), and wild-type mice on chow (small white squares) and HFD (large white squares). IPGTTs were performed after a $16 \mathrm{~h}$ fast (overnight) and IPITTs after a $6 \mathrm{~h}$ fast. Glucose $(2 \mathrm{mg} / \mathrm{g})$ and insulin $(0.75$ units $/ \mathrm{kg})$ were injected intraperitoneally and glycaemia was measured at different times after injection. IPGTTs were performed on mice at 12 weeks of age (same result was obtained with older mice, up to 19 weeks old) and IPITTs on mice of 20 and 21 weeks of ages in HFD and chow diet groups respectively. $n=5-7$ for each group, ${ }^{*} p<0.05$. c, d Insulin signalling is not altered in skeletal muscle of chow-fed MLC/Socs-3 transgenic (tg, black bars) male mice compared with wild-type (wt, white bars) littermates. EDL from males (22 weeks) were stimulated or not ex vivo with insulin $(1 \mathrm{nmol} / \mathrm{l})$ for $30 \mathrm{~min}$. Total lysates and lysates subjected to immunoprecipitation (IP) with a flag antibody or an IRS antibody were separated by SDS-PAGE and analysed with anti-p85, anti-phospho-GSK- $3 \alpha / \beta$, anti-GSK-3 $\beta$, anti-phospho-PKB, anti-PKB and anti-SOCS-3 antibodies. A representative western blot (c) is shown, with quantification of the bands (d) from three different experiments. e, f Affymetrix and r-t PCR analyses were performed on wild-type (white bars) and transgenic (black bars) tibialis muscle (wild-type $n=3$, transgenic $n=3$ ). For r-t PCR, amplifications were repeated in EDL muscle of the same group of animals and in tibialis of two other groups of animals (wild-type $n=4$, transgenic $n=3$ for each group). Results of a representative r-t PCR are shown. ${ }^{*} p \leq 0.05 ;{ }^{* * *} p \leq 0.001$ mice on HFD (Fig. 3b). Importantly, the decreased insulin sensitivity was not observed in a group of transgenic animals with body weight similar to wild-type (ESM Fig. 2a).

To summarise, compared with wild-type, MLC/Socs-3 mice on a HFD developed an exacerbated organismal insulin resistance, which appears to be due to their increased obesity.
MLC/Socs-3 mice have unaltered insulin signalling in their skeletal muscle

Since it has been shown that SOCS-3 inhibits insulin signalling in vitro [2], we sought to evaluate whether this also occurred in the muscle of our transgenic animals. However, we did not detect changes in insulin-induced IRS/ p85 co-immunoprecipitation and in PKB and GSK-3 
activation in Socs-3 transgenic mice compared with wildtype (Fig. 3c, d). Consistent with the unaltered insulin signalling in transgenic muscle, glycogen synthesis in response to insulin was similar in wild-type and transgenic animals (ESM Fig. 2b). To summarise, in mice constitutive SOCS-3 production in skeletal muscle failed to alter insulin signalling in this tissue.

To define more precisely the molecular changes that could explain the maintenance of insulin signalling in Socs-3 transgenic muscle, we performed an Affymetrix analysis on MLC/Socs-3 and wild-type mice. Generally speaking, a series of genes with modest variation in their expression level was revealed (for complete data see GEO website; www.ncbi.nlm.nih.gov/geo/query/acc.cgi?token=jpwtxqiea ucgihm\&acc=GSE17063, accessed 20 July 2009). Using r-t PCR, we confirmed the most relevant gene variations in tibialis and EDL muscles, and in three different groups of animals. Some of the most interesting observations are shown in Fig. 3e and f. Notably, we found that both Socs-2 and Cis (also known as Cish) had a threefold reduction in their expression (e). Cntfr expression was increased (about threefold), whereas $L p l$ mRNA was reduced (1.42-fold) in Socs-3 transgenic muscle (f).

Potential role of SOCS-3 in interfering with muscle calcineurin and in reducing locomotor activity

Our Affymetrix analysis revealed a set of genes that was deregulated in skeletal muscle from Socs-3 transgenic animals. Interestingly, some of them operate downstream of the phosphatase calcineurin, i.e. $\mathrm{Lpl}$ and Smtck [27, 28]. Moreover, data obtained in $\mathrm{T}$ cells demonstrated an interaction between calcineurin A and GST-SOCS-3 protein [17]. We next examined whether a similar interaction could occur in muscle of MLC/Socs-3 mice. In brief, using whole-muscle lysate from wild-type animals, we performed pull-down with GST-SOCS-3 (vs GST alone). Proteomic analysis of proteins specifically associated with GSTSOCS-3 revealed calcineurin A (Fig. 4a). This interaction was confirmed by western blotting analysis (Fig. 4b).

Using immunohistochemistry we looked at calcineurin localisation inside skeletal muscle. Consistent with previously published data [29], the calcineurin immunohistochemical signal exhibited a pattern formed by discrete rows of puncta that run transversally to the myofibril (Fig. 4c). Remarkably, in transgenic animals, calcineurin was delocalised to the periphery of the fibres and the size of puncta positively stained for calcineurin was increased. Interestingly, immunostaining for calcineurin A and SOCS-3 showed co-localisation of calcineurin with SOCS-3 in transgenic animals (Fig. 4d). Moreover, co-localisation between calcineurin and SOCS-3 occurred mainly in areas where the calcineurin signal was perturbed. Finally, calcineurin protein level was unaltered in transgenic muscle (ESM Fig. 3). Taken together, our results suggest that constitutively produced SOCS-3 interacts with calcineurin A in skeletal muscle in vivo, inducing its sequestration and hence potentially altering its biological functions.

Constitutive SOCS-3 production changes muscle morphology

Calcineurin has been shown to be a key actor in the switch of type II muscular fibres to type I, improving muscle oxidative characteristics $[27,28,30]$. Thus, using in situ staining of SDH activity, we investigated the effect of constitutive SOCS-3 production on fibre distribution in tibialis anterior. As shown in Fig. 5a, constitutive SOCS-3 production had no detectable effect on fibre number in transgenic compared with wild-type mice and did not change the percentage of SDH-positive fibres.

Calcineurin is also implicated in the calcium fluxes involved in muscle contractility. Using transmission electron microscopy, we compared skeletal muscle morphology of transgenic mice with wild-type. Interestingly, Socs-3 transgenic muscles showed a specific dilatation of the cistern of sarcoplasmic reticulum with no modification of the T-tubule structure (Fig. 5b). Moreover, mitochondria in transgenic muscle were frequently abnormally shaped, larger in size and of translucent appearance (Fig. 5c). In line with mitochondrial alteration in Socs-3 transgenic muscle, our Affymetrix and r-t PCR investigations revealed decreased expression of the mitochondrial markers, Smtck (1.41 fold) and Slc25a3 (2.16-fold) (Fig. 5d).

Given the crucial role of calcineurin and sarcoplasmic reticulum in skeletal muscle contraction, we studied whether constitutive production of SOCS-3 could impact on locomotor activity. First, our analysis of longitudinal movements suggested that the activity was significantly diminished in transgenic mice (14.1\% day, $13.7 \%$ night) (Fig. 6a). To determine whether the transgenic animals have perturbed locomotion, we estimated voluntary exercise of wild-type and transgenic mice with similar weight using wheel-coupled cages. We found that the transgenic animals had reduced spontaneous physical activity. Moreover, this decrease was exacerbated within 1 week of training (Fig. 6b).

\section{Discussion}

Several in vitro and in vivo studies have suggested that SOCS3 is implicated in development of insulin resistance and type 2 diabetes [3, 31-33]. However, it is still not clear whether increased SOCS-3 levels in vivo are a cause or rather a consequence of insulin resistance. In addition, SOCS-3 appears to affect signalling pathways differently depending on the tissue in which its production is increased $[10,12]$. 
Fig. 4 Potential regulation of calcineurin functions by SOCS3. a, b A GST pull-down analysis was performed using GST alone vs GST/SOCS-3 and whole-cell lysate (WCL) from wild-type gastrocnemius. Proteomic (a) and western blot (b) analyses revealed an interaction between GST/SOCS-3 and calcineurin $(\mathrm{CnA} \alpha / \beta)$. MW, molecular weight. c, d Tibialis anterior muscle from 15-weekold wild-type (wt, $n=5$ ) or transgenic (tg, $n=5)$ mice were fixed in paraformaldehyde and embedded in paraffin. Immunohistochemistry was performed using anti-calcineurin and antiSOCS-3 antibodies. For nuclear counterstaining ToPro 3 iodide was used. White arrows (c) point to the calcineurin targeting to fibre periphery in transgenic animals (top, scale bars: $40 \mu \mathrm{m}$ ) and the enlarged puncta staining of calcineurin in transgenic animals (bottom, scale bars:

$12 \mu \mathrm{m})$. d White arrows point to the co-localisation of calcineurin and SOCS-3 in enlarged puncta. Scale bar, top: $30 \mu \mathrm{m}$; bottom: $20 \mu \mathrm{m}$ a

\begin{tabular}{|c|c|c|}
\hline & GST & GST/SOCS-3 \\
\hline $\mathrm{CnA} \alpha / \beta$ & - & + \\
\hline
\end{tabular}

Proteomics
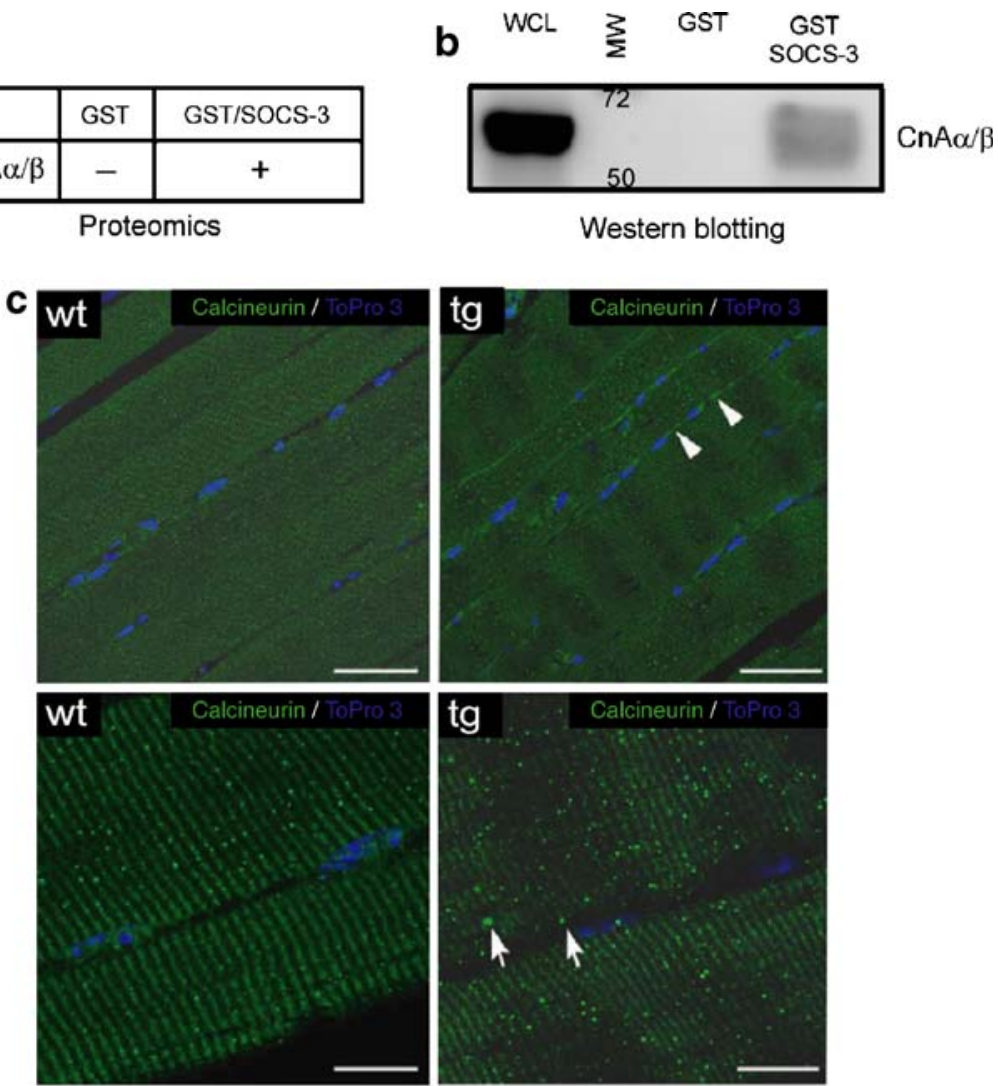

Western blotting
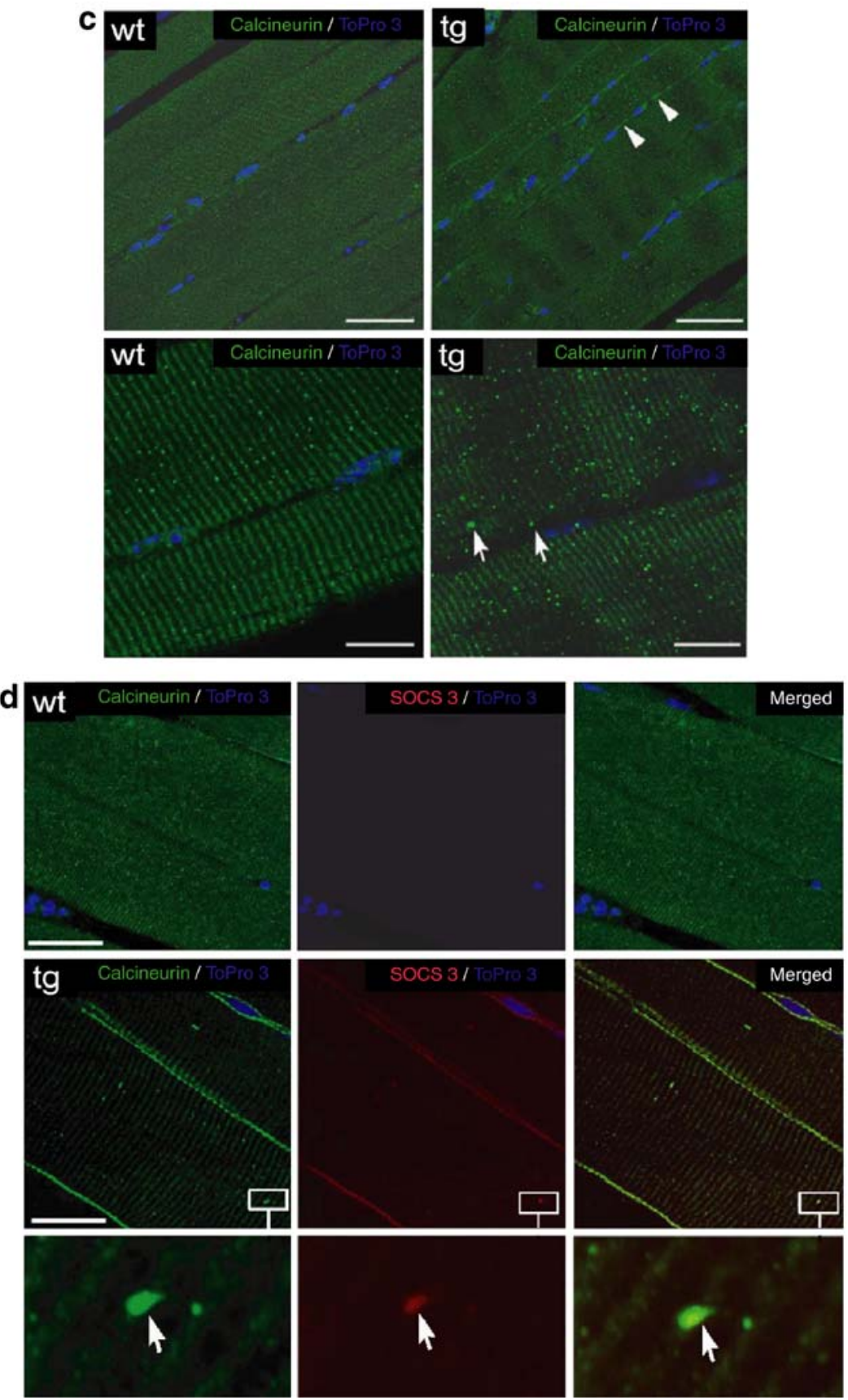

Considering the crucial contribution of muscle to glucose homeostasis, we investigated the role of SOCS-3 in skeletal muscle of intact animals using tissue-specific transgenic mice.

The most striking observation of our study is the pronounced obese phenotype of our transgenic mice. Their overweight is present on a standard chow diet, but comes to full bloom on a HFD. All white adipose depots analysed were enlarged in transgenic mice leading to a twofold increase in total white adipose mass. We further found that our Socs-3 transgenic mice were very susceptible to develop HFD-induced insulin resistance. However, this 


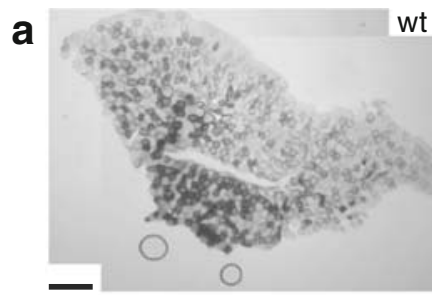

Total

$\% \mathrm{SDH}+$

$\% \mathrm{SDH}-$

b)
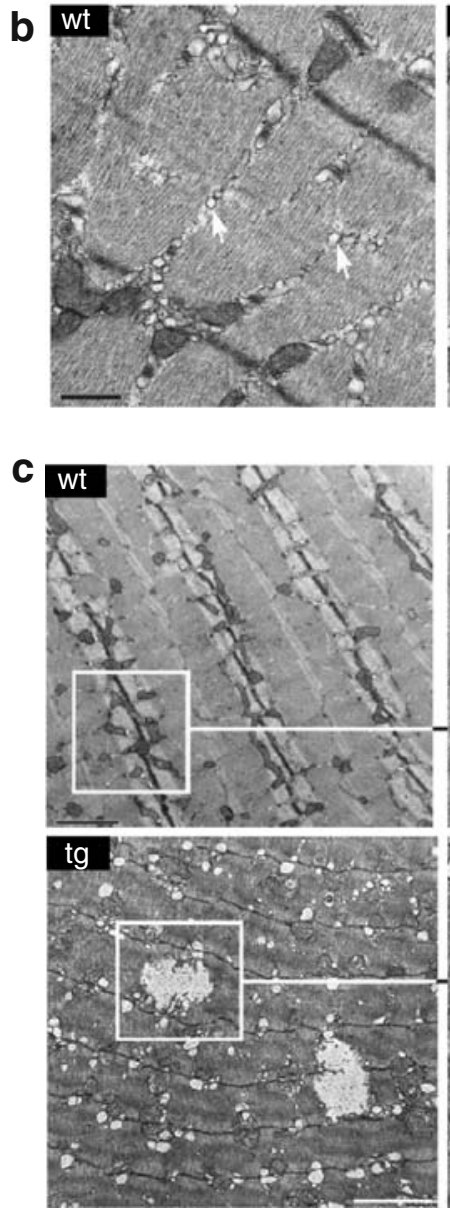

d

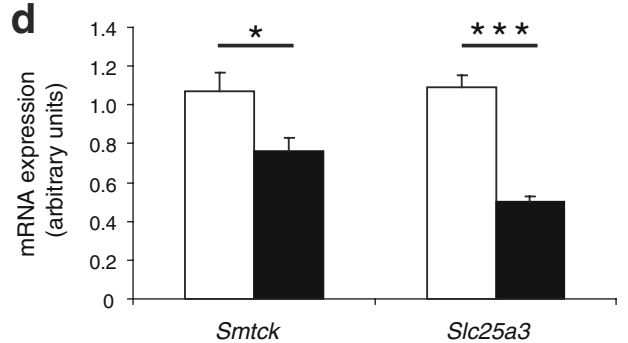

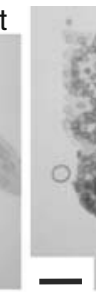

wt
$55.4 \pm 3.8$

$44.6 \pm 3.8$

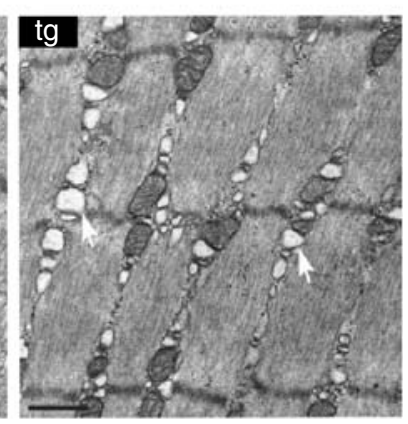

$\operatorname{tg}$

$1,534 \pm 142$

$53.6 \pm 3.4$

$46.4 \pm 3.4$
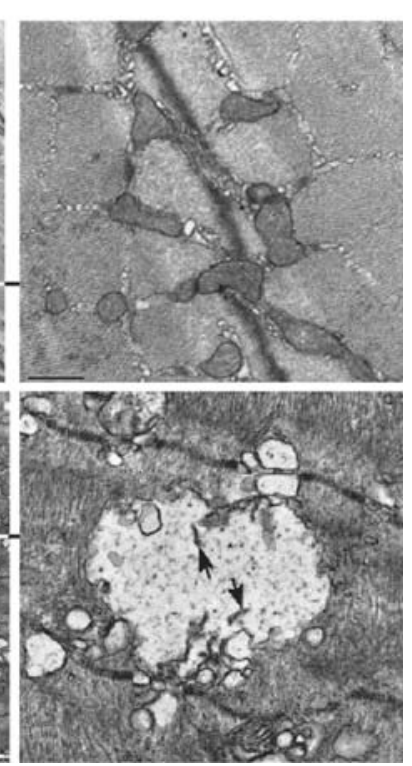

Fig. 5 a Constitutive production of SOCS-3 in skeletal muscle does not change the amount of SDH-positive fibres in tibialis anterior. Tibialis anterior muscle from 24-week-old wild-type (wt) or transgenic (tg) mice were cryosectioned and stained for SDH activity. Total number of fibres was determined on serial sections. Values are means \pm SEM from three animals of each genotype. Scale bar: $1 \mathrm{~mm}$. b, c SOCS-3 constitutive production alters skeletal muscle ultra structure. Tibialis anterior muscle from 15 -week-old wild-type (wt, $n=5$ ) or transgenic (tg, $n=5$ ) mice were fixed in glutaraldehyde/paraformaldehyde and embedded in epoxy resin. Ultrathin sections stained with lead citrate were examined by transmission electron microscopy. Representative pictures are shown. White arrows (b), sarcoplasmic reticulum; black scale bar: $0.5 \mu \mathrm{m}$. Black arrows (c), altered mitochondria pattern; white scale bar: $1 \mu \mathrm{m}$; black scale bars: $2 \mu \mathrm{m}$ (wt, left) and $0.5 \mu \mathrm{m}$ (wt, right). d Decreased expression of mitochondrial markers in MLC/Socs-3 skeletal muscle. Affymetrix and r-t PCR analyses were performed on wild-type (white bars) and transgenic (black bars) muscle (wild-type $n=3$, transgenic $n=3$ ). Results of a representative r-t PCR are shown. ${ }^{*} p \leq 0.05 ;{ }^{* * *} p \leq 0.001$

increased insulin resistance seems to be due to the exacerbated obesity of transgenic mice and not directly to the chronic production of SOCS-3 in muscle. In line with this fact, constitutive SOCS-3 production does not appear to affect insulin action in muscle when measured acutely. At first glance this observation is counter-intuitive, as several studies, including our own, have shown that increased SOCS-3 levels results in decreased insulin signalling in the tissue concerned [2, 3]. One of the important differences between our transgenic mice and the models used in the above-mentioned studies is the presence of chronic vs acute SOCS production. Obviously prolonged SOCS production is more likely to lead to compensatory mechanisms aimed at maintaining homeostasis, such as the reduction of other negative regulators of insulin action. In fact, such a scenario might be occurring in our transgenic mice, where, using Affymetrix and r-t PCR analyses, we found that constitutive SOCS-3 production in muscle resulted in decreased expression of several modulators of insulin signalling. Thus, a significant decrease in Socs-2 and $C i$ is was found. So far, SOCS-2 and CIS have been implicated mainly in regulating postnatal growth [34, 35] and very little is known about their role in skeletal muscle metabolism. However, very recent data strongly suggest that SOCS-2 is a key actor in modulating insulin signal in muscle. Indeed, it has been shown that chemokine CXC ligand-5 induces insulin resistance mainly by inhibiting insulin signalling in muscle, which may be driven by SOCS-2 production [36]. Moreover, a given SOCS protein appears to promote the proteasomal degradation of other SOCS proteins [37]. This suggests that SOCS proteins are able to transregulate the expression of other members of the family, leading to auto-regulatory compensatory mechanisms. Such a scenario could counterbalance the effects of increased SOCS-3 levels, hence explaining the unchanged insulin signal in transgenic skeletal muscle, despite constitutive SOCS-3 production. 

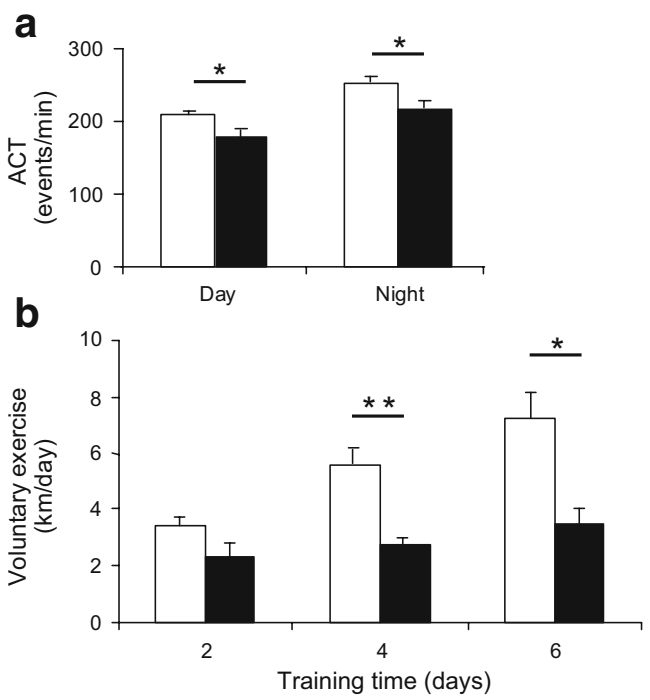

Fig. 6 Decreased locomotor activity in MLC/Socs-3 mice. a Measurement of locomotor activity (Act) was performed over a $24 \mathrm{~h}$ period and data were averaged for each mouse (wild-type, white bars, $n=7$; transgenic, black bars, $n=6$ ). b After 3 days of accommodation, voluntary exercise was monitored for 6 days. The number of quarter wheel-turns was measured every day for each mouse ( $n=5$ per group). Distance $\operatorname{run}(\mathrm{km})=($ quarter wheel-turns $/ 4) \times(2 \pi \mathrm{r}) . \quad * p \leq 0.05$; $* * p \leq 0.01$

Another candidate for maintaining insulin signalling in our transgenic mice was revealed by Affymetrix analysis. Indeed, the mRNA of Cntfr was increased in muscle from transgenic animals. Previous data have strongly suggested a role for ciliary neurotrophic factor (CNTF) in insulin resistance and obesity [38, 39]. CNTF acts centrally and peripherally; remarkably, it appears to reduce inflammatory signalling cascades associated with lipid accumulation in liver and muscle. By doing so, CNTF is thought to maintain or restore insulin sensitivity in peripheral tissues.

Finally, we found a decrease in Lpl mRNA level in Socs-3 transgenic muscle. In line with the observations made in our Socs-3 transgenic model, old and HFD-fed $\mathrm{Smlp}^{/-}$mice developed obesity associated with systemic insulin resistance [40]. Moreover, the same study shows that $\mathrm{Lpl}$ deletion in skeletal muscle increased insulin signalling in this tissue.

Interestingly, $\mathrm{Lpl}$ is one of the genes regulated by the calcineurin/NFAT pathway and drives part of the biological effects of calcineurin [27]. Moreover, previous studies in $\mathrm{T}$ cells have shown that SOCS-3 is able to interact with calcineurin A, hampering the signal downstream of calcineurin [17]. Using a GST-pull down analysis, we demonstrated that calcineurin A from muscle interacts with a GST-SOCS-3 fusion protein, suggesting that such an interaction may in fact occur in Socs-3 transgenic muscle. Indeed, as shown with our immunohistochemical analysis, calcineurin co-localised perfectly with SOCS-3 in transgenic muscle. In addition, in transgenic mice, calcineurin seems to be targeted to the periphery of the fibres and increased punctated staining occurs. This suggests that by sequestering calcineurin, SOCS-3 could alter its biological function. Albeit controversial, calcineurin is thought to be involved in transforming the fast-twitch skeletal muscle fibres to a slow-twitch phenotype [41, 42]. In particular, it has been shown that production of constitutively active Calcineurin A $\left(\mathrm{CnA}^{*}\right)$ in skeletal muscle leads to increased oxidative metabolism [27, 28]. Consistent with these results, a reduction in oxidative characteristics has been shown in calcineurin $A$ gene-targeted mice [43]. The ability of calcineurin to modulate skeletal muscle metabolism seems to be due mainly to the regulation of specific target genes implicated in glucose (e.g. Gapdh, Pdk4) and lipid (e.g. Lpl, CD36) metabolism [27]. By Affymetrix analysis and r-t PCR, we found that $L p l$ and Smtck gene expression are decreased in MLC/Socs-3 mice, whereas they are increased in $\mathrm{CnA}^{*}$ mice. However, using $\mathrm{SDH}$ staining, we failed to detect alterations of fibre distribution (oxidative/glycolytic) in skeletal muscle, suggesting that the deleterious effects of SOCS-3 on calcineurin must affect another function of the phosphatase. In addition to its role in muscle oxidative pathway, calcineurin is thought to be involved in muscle contraction and relaxation. The sarcoplasmic reticulum is clearly implicated in $\mathrm{Ca}^{2+}$ fluxes into muscle cells [44, 45]. Membrane depolarisation is detected by the voltage-sensing dihydropyridine-sensitive L-type calcium channel (DHPR), which induces calcium release from the sarcoplasmic reticulum through the ryanodine receptors (RyR), leading to muscle contraction. Note that calcineurin co-localises with RyR and DHPR in mature skeletal muscle [29]. The association between calcineurin and RyR is $\mathrm{Ca}^{2+}$-dependent and regulates, via dephosphorylation, the activity of RyR and the subsequent $\mathrm{Ca}^{2+}$ release by sarcoplasmic reticulum [18]. Using transmission electron microscopy, we found altered sarcoplasmic reticulum and mitochondria patterns in transgenic muscle. It has to be stressed that the dilatation of sarcoplasmic reticulum associated with swollen mitochondria is pronounced in type II fibres, whereas it is more discrete in type I (data not shown). Thus, these alterations are most likely to be linked to robust Socs-3 transgene expression, which is mainly present in type II fibres. We further showed that expression of Smtck and Slc25a3 was reduced in MLC/Socs-3 muscles. Interestingly, these two mitochondrial markers have been implicated in muscular tonicity [46, 47]. Altogether our observations argue for the occurrence of SOCS-3-induced mitochondrial dysfunction in the transgenic mice. Thus, even though our SDH analysis did not detect differences in distribution of type I and type II fibres, we cannot exclude the possibility that the Socs-3 transgenic animals have a modified oxidative activity in muscle, which could impact on organismal metabolism and/ or physical activity. 
The molecular and morphological alterations discussed above have often been found to be associated with decreased locomotor activity [47, 48]. As expected, we revealed reduced locomotor activity in Socs-3 transgenic animals. It is important to stress that the analysis of voluntary exercise was performed using wild-type and transgenic animals with similar body weight. Thus, our results clearly demonstrate that the overweight of transgenic mice is a consequence of the locomotor defect and not a cause of it.

Due to their regulatory role in several signalling pathways, the SOCS proteins have been put forward as potential key therapeutic targets. However, the exact role of SOCS in vivo has to be defined using highly relevant animal models. The present study brings new insight into the role of SOCS3 in skeletal muscle. Our first key finding is that, as demonstrated in fat-specific transgenic mice, SOCS-3 constitutive production in skeletal muscle is not in itself sufficient to induce the establishment of organismal metabolic disorders such as diabetes. However, in contrast to what was described for adipose tissue, chronic SOCS-3 production in muscle modulates the expression of several genes coding for regulatory proteins, which may explain the maintenance of normal insulin sensitivity in skeletal muscle. Our second major contribution is that we reveal a novel function of SOCS-3 in muscle physiology, which impacts on locomotor activity. The data suggest that constitutive production of SOCS-3 in skeletal muscle alters mitochondrial integrity and the excitation-contraction coupling, leading to muscle fatigability. Our urgent challenge is now to identify the precise mechanisms by which the SOCS proteins control these fundamental functions of muscle metabolism.

Acknowledgements The expertise of A. Desquesnes from the Functional Exploration Platform of Toulouse Midi-Pyrénées Génopôle is acknowledged. We are grateful to C. Hinault, N. Gautier and C. Gaudel for expertise with ex vivo muscle experiments. We thank L. Moya and D. Ramos for technical assistance in transmission electron microscopy and immunohistochemical analysis. Microarray experiments were carried out by the MarseilleNice-Sophia Antipolis Génopôle Platform; we thank in particular P. Barbry and C. Moreilhon-Brest. We are grateful to J.-M. Guigonis for his expertise in proteomic analysis and to J. Neels for constructive comments. E. Cognard was supported by a grant from FRM (Fondation pour la Recherche Médicale, Paris, France) and P. Lebrun by a grant from the EFSD/MSD programme (20052007). Our research in Nice was supported by INSERM, Université de Nice-Sophia Antipolis, le Conseil Général des Alpes-Maritimes, le Conseil Régional PACA and the European Community (Grant FP6 Eugene2 [LSHM-CT-2004-512013]). Research work performed in F. Bosch laboratory was supported by a grant from Plan Nacional I+D+I (SAF2005-01262), Spain and by Grant FP6 Eugene2 (LSHM-CT-2004-512013).

Duality of interest The authors declare that there is no duality of interest associated with this manuscript.

\section{References}

1. Fujimoto M, Naka T (2003) Regulation of cytokine signalling by SOCS family molecules. Trends Immunol 24:659-666

2. Emanuelli B, Peraldi P, Filloux C, Sawka-Verhelle D, Hilton D, Van Obberghen E (2000) SOCS-3 is an insulin-induced negative regulator of insulin signaling. J Biol Chem 275:15985-15991

3. Ueki K, Kondo T, Kahn CR (2004) Suppressor of cytokine signaling 1 (SOCS-1) and SOCS-3 cause insulin resistance through inhibition of tyrosine phosphorylation of insulin receptor substrate proteins by discrete mechanisms. Mol Cell Biol 24:5434-5446

4. Rui L, Yuan M, Frantz D, Shoelson S, White MF (2002) SOCS-1 and SOCS-3 block insulin signaling by ubiquitin-mediated degradation of IRS1 and IRS2. J Biol Chem 277:42394-42398

5. Bjorbak C, Lavery HJ, Bates SH et al (2000) SOCS3 mediates feedback inhibition of the leptin receptor via Tyr985. J Biol Chem 275:40649-40657

6. Dunn SL, Bjornholm M, Bates SH, Chen Z, Seifert M, Myers MG (2005) Feedback inhibition of leptin receptor/Jak2 signaling via Tyr1138 of the leptin receptor and suppressor of cytokine signaling 3. Mol Endocrinol 19:925-938

7. Howard JK, Cave BJ, Oksanen LJ, Tzameli I, Bjorbaek C, Flier JS (2004) Enhanced leptin sensitivity and attenuation of dietinduced obesity in mice with haploinsufficiency of Socs3. Nat Med 10:734-738

8. Kievit P, Howard JK, Badman MK et al (2006) Enhanced leptin sensitivity and improved glucose homeostasis in mice lacking suppressor of cytokine signaling-3 in POMC-expressing cells. Cell Metab 4:123-132

9. Ueki K, Kadowaki T, Kahn CR (2005) Role of suppressors of cytokine signaling SOCS-1 and SOCS-3 in hepatic steatosis and the metabolic syndrome. Hepatol Res 33:185-192

10. Ueki K, Kondo T, Tseng YH, Kahn CR (2004) Central role of suppressors of cytokine signaling proteins in hepatic steatosis, insulin resistance, and the metabolic syndrome in the mouse. Proc Natl Acad Sci U S A 101:10422-10427

11. Shi H, Cave B, Inouye K, Bjorbaek C, Flier JS (2006) Overexpression of suppressor of cytokine signaling 3 in adipose tissue causes local but not systemic insulin resistance. Diabetes 55:699-707

12. Lindberg K, Ronn SG, Tornehave D et al (2005) Regulation of pancreatic beta-cell mass and proliferation by SOCS-3. J Mol Endocrinol 35:231-243

13. Ronn SG, Börjesson A, Bruun C et al (2008) Suppressor of cytokine signalling-3 expression inhibits cytokine-mediated destruction of primary mouse and rat pancreatic islets and delays allograft rejection. Diabetologia 51:1873-1882

14. Spangenburg EE (2005) SOCS-3 induces myoblast differentiation. J Biol Chem 280:10749-10758

15. Spangenburg EE, Brown DA, Johnson MS, Moore RL (2006) Exercise increases SOCS-3 expression in rat skeletal muscle: potential relationship to IL-6 expression. J Physiol 572:839-848

16. Fasshauer M, Klein J, Lossner U, Paschke R (2002) Isoproterenol is a positive regulator of the suppressor of cytokine signaling-3 gene expression in 3T3-L1 adipocytes. J Endocrinol 175:727-733

17. Banerjee A, Banks AS, Nawijn MC, Chen XP, Rothman PB (2002) Cutting edge: suppressor of cytokine signaling 3 inhibits activation of NFATp. J Immunol 168:4277-4281

18. Shin DW, Pan Z, Bandyopadhyay A, Bhat MB, Kim DH, Ma J (2002) $\mathrm{Ca}(2+)$-dependent interaction between FKBP12 and calcineurin regulates activity of the $\mathrm{Ca}(2+)$ release channel in skeletal muscle. Biophys J 83:2539-2549

19. Rosenthal N, Kornhauser JM, Donoghue M, Rosen KM, Merlie JP (1989) Myosin light chain enhancer activates muscle-specific, developmentally regulated gene expression in transgenic mice. Proc Natl Acad Sci U S A 86:7780-7784 
20. Otaegui PJ, Ferre T, Riu E, Bosch F (2003) Prevention of obesity and insulin resistance by glucokinase expression in skeletal muscle of transgenic mice. FASEB J 17:2097-2099

21. Heydrick SJ, Jullien D, Gautier N et al (1993) Defect in skeletal muscle phosphatidylinositol-3-kinase in obese insulin-resistant mice. J Clin Invest 91:1358-1366

22. Hinault C, Mothe-Satney I, Gautier N, Lawrence JC Jr, Van Obberghen E (2004) Amino acids and leucine allow insulin activation of the $\mathrm{PKB} / \mathrm{mTOR}$ pathway in normal adipocytes treated with wortmannin and in adipocytes from $\mathrm{db} / \mathrm{db}$ mice. FASEB J 18:1894-1896

23. Smyth GK, Michaud J, Scott H (2005) The use of within-array replicate spots for assessing differential expression in microarray experiments. Bioinformatics 21:2067-2075

24. Cayatte C, Pons C, Guigonis JM et al (2006) Protein profiling of rat ventral prostate following chronic finasteride administration: identification and localization of a novel putative androgenregulated protein. Mol Cell Proteomics 5:2031-2043

25. Luquet S, Lopez-Soriano J, Holst D et al (2003) Peroxisome proliferator-activated receptor delta controls muscle development and oxidative capability. FASEB J 17:2299-2301

26. Bauche IB, El Mkadem SA, Pottier AM et al (2007) Overexpression of Adiponectin targeted to adipose tissue in transgenic mice: impaired adipocyte differentiation. Endocrinology 148:1539-1549

27. Naya FJ, Mercer B, Shelton J, Richardson JA, Williams RS, Olson EN (2007) Calcineurin regulates skeletal muscle metabolism via coordinated changes in gene expression. J Biol Chem 282:1607-1614

28. Long YC, Glund S, Garcia-Roves PM, Zierath JR (2000) Stimulation of slow skeletal muscle fiber gene expression by calcineurin in vivo. J Biol Chem 275:4545-4548

29. Torgan CE, Daniels MP (2006) Calcineurin localization in skeletal muscle offers insights into potential new targets. J Histochem Cytochem 54:119-128

30. Long YC, Zierath JR (2008) Influence of AMP-activated protein kinase and calcineurin on metabolic networks in skeletal muscle. Am J Physiol Endocrinol Metab 295:E545-E552

31. Emanuelli B, Peraldi P, Filloux C et al (2001) SOCS-3 inhibits insulin signaling and is up-regulated in response to tumor necrosis factor-alpha in the adipose tissue of obese mice. J Biol Chem 276:47944-47949

32. Rieusset J, Bouzakri K, Chevillotte E et al (2004) Suppressor of cytokine signaling 3 expression and insulin resistance in skeletal muscle of obese and type 2 diabetic patients. Diabetes 53:22322241

33. Gylvin T, Nolsoe R, Hansen T et al (2004) Mutation analysis of suppressor of cytokine signalling 3 , a candidate gene in type 1 diabetes and insulin sensitivity. Diabetologia 47:1273-1277
34. Metcalf D, Greenhalgh CJ, Viney E et al (2000) Gigantism in mice lacking suppressor of cytokine signalling-2. Nature 405:1069-1073

35. González L, Miquet JG, Sotelo AI, Bartke A, Turyn D (2002) Cytokine-inducible $\mathrm{SH} 2$ protein up-regulation is associated with desensitization of GH signaling in GHRH-transgenic mice. Endocrinology 143:386-394

36. Chavey C, Lazennec G, Lagarrigue S et al (2009) CXC Ligand 5 is an adipose-tissue derived factor that links obesity to insulin resistance. Cell Metabolism 9:339-349

37. Piessevaux J, Lavens D, Montoye T et al (2006) Functional crossmodulation between SOCS proteins can stimulate cytokine signaling. J Biol Chem 281:32953-32966

38. Watt MJ, Hevener A, Lancaster GI, Febbraio MA (2006) Ciliary neurotrophic factor prevents acute lipid-induced insulin resistance by attenuating ceramide accumulation and phosphorylation of cJun N-terminal kinase in peripheral tissues. Endocrinology 147:2077-2085

39. Watt MJ, Dzamko N, Thomas WG et al (2006) CNTF reverses obesity-induced insulin resistance by activating skeletal muscle AMPK. Nat Med 12:541-548

40. Wang H, Knaub LA, Jensen DR et al (2009) Skeletal musclespecific deletion of lipoprotein lipase enhances insulin signaling in skeletal muscle but causes insulin resistance in liver and other tissues. Diabetes 58:116-124

41. Bigard X, Sanchez H, Zoll J et al (2000) Calcineurin co-regulates contractile and metabolic components of slow muscle phenotype. J Biol Chem 275:19653-19660

42. Swoap SJ, Hunter RB, Stevenson EJ et al (2000) The calcineurinNFAT pathway and muscle fiber-type gene expression. Am J Physiol Cell Physiol 279:C915-C924

43. Parsons SA, Wilkins BJ, Bueno OF, Molkentin JD (2003) Altered skeletal muscle phenotypes in calcineurin Aalpha and Abeta genetargeted mice. Mol Cell Biol 23:4331-4343

44. Rossi AE, Dirksen RT (2006) Sarcoplasmic reticulum: the dynamic calcium governor of muscle. Muscle Nerve 33:715-731

45. Bellinger AM, Reiken S, Dura M et al (2008) Remodeling of ryanodine receptor complex causes "leaky" channels: a molecular mechanism for decreased exercise capacity. Proc Natl Acad Sci U S A 105:2198-2202

46. Palmieri F (2004) The mitochondrial transporter family (SLC25): physiological and pathological implications. Pflugers Arch 447:689709

47. Momken I, Lechêne P, Koulmann N et al (2005) Impaired voluntary running capacity of creatine kinase-deficient mice. J Physiol 15:951964

48. Reggiani C, te Kronnie T (2006) RyR isoforms and fibre type-specific expression of proteins controlling intracellular calcium concentration in skeletal muscles. J Muscle Res Cell Motil 27:327-335 